10,05

\title{
Ab initio и экспериментальное исследование колебательных свойств кристаллов $\mathrm{TIFeS}_{2}$ и $\mathrm{TIFeSe}_{2}$
}

\author{
(C) З.А. Джахангирли ${ }^{1,2}$, Р.Г. Велиев ${ }^{1}$, И.А. Мамедова ${ }^{1}$, З.И. Бадалова ${ }^{1}$, \\ Д.А. Мамедов ${ }^{1}$, Н.Т. Мамедов ${ }^{1}$, Н.А. Абдуллаев ${ }^{1,2, \text { ฯ }}$ \\ ${ }^{1}$ Институт ффизики Национальной академии наук Азербайджана, \\ AZ1143 Баку, Азербайджан \\ 2 Бакинский государственный университет, \\ AZ1148 Баку, Азербайджан \\ 『E-mail:abnadir@mail.ru
}

Поступила в Редакцию 27 апреля 2021 г.

В окончательной редакции 27 апреля 2021 г.

Принята к публикации 7 мая 2021 г.

Решеточные колебательные свойства кристаллов $\mathrm{TlFeS}_{2}$ и $\mathrm{TlFeSe}_{2}$ исследованы экспериментально с использованием комбинационного рассеяния (КР) и инфракрасного отражения (ИК) света, а также теоретически с использованием теории функционала плотности (DFT). Полное колебательное представление, составленное на основе анализа фактор групп симметрии, содержит 12 КР активных мод и 9 ИК активных мод. Из них экспериментально обнаружены и идентифицированы 6 КР активных и 3 ИК активных мод для $\mathrm{TlFeS}_{2}$, и 4 KР активных 3 ИК активных мод для $\mathrm{TlFeSe}_{2}$.

Ключевые слова: оптические фононы, динамика решетки, первопринципные вычисления, группа симметрии, фононные спектры, дисперсия фононов.

DOI: 10.21883/FTT.2021.10.51416.099

\section{1. Введение}

$\mathrm{TlFeS}_{2}$ известен как кристаллоструктурный аналог природного минерала „рагинит“ $[1]$, обладающего характерными прямолинейными тетраэдрическими $\mathrm{FeS}_{4}$ цепочками, то есть в структуре $\mathrm{TlFeS}_{2}$ трехвалентный атом железа, связанный с четырьмя атомами серы посредством сил ковалентной химической связи, образует тетраэдр, в центре которого расположен катион железа, а в вершинах расположены анионы серы. Эти $\mathrm{Fe}^{3+} \mathrm{S}_{4}^{2-}$ тетраэдры, сочлененные общими ребрами, образуют цепочки вытянутые вдоль кристаллографической оси $c$, а одновалентные атомы таллия $\mathrm{Tl}$ располагаются слоями в тригонально-призматических пустотах кристаллической структуры вдоль кристаллографической оси $a$ псевдотетрагональной призмы $\mathrm{TlFeS}_{2}$ с моноклинной сингонией. В пространственном кристаллоструктурном строении соединения $\mathrm{TlFeS}_{2}$, слоевые пакеты катионов таллия взаимодействуют с цепочками тетраэдров посредством сил ионной химической связи. Между тетраэдрическими цепочками и между слоями из катионов таллия действуют слабые связи ван-дер-ваальсового типа.

Отметим, что вышеизложенное относится и к $\mathrm{TlFeSe}_{2}$, поскольку $\mathrm{TlFeS}_{2}, \mathrm{TlFeSe}_{2}$ изоструктурны [2] и кристаллическая структура этих соединений предполагает сильную зависимость их физических свойств от основных кристаллографических направлений, в частности магнитноактивные свойства $\mathrm{TlFeS}_{2}, \mathrm{TlFeSe}_{2}$ проявляются вдоль оси $c$ [3], то есть вдоль цепи тетраэдров по линейным цепочкам ионов $-\mathrm{Fe}^{3+}-X^{2-}-\mathrm{Fe}^{3+}-$
$(X$ - халькоген, $\mathrm{S}$ или $\mathrm{Se})$. По цепочкам же ионов $-\mathrm{Fe}^{3+}-X^{2-}-\mathrm{Tl}^{1+}-X^{2-}-\mathrm{Fe}^{3+}-$, вытянутых вдоль красталлографической оси $a$ кристаллической структуры соединений $\mathrm{TlFeS}_{2}$ и $\mathrm{TlFeSe}{ }_{2}$, которые примерно перпендикулярны цепи тетраэдров $\mathrm{Fe} X_{4}$, магнитноактивные свойства $\mathrm{TlFeS}_{2}, \mathrm{TlFeSe}_{2}$ не проявляются [3].

Следует отметить, что выводы о природе магнитного фазового превращения в $\mathrm{TlFeS}_{2}, \mathrm{TlFeSe}_{2}$ весьма противоречивы [1,3-10]. По нашему мнению, магнитная структура полупроводников $\mathrm{TlFeS}_{2}$ и $\mathrm{TlFeSe}_{2}$ квазиодномерноантиферромагнитная [11]. По-видимому, авторы вышеперечисленных работ при интерпретации экспериментальных результатов, не акцентировались на кристаллоструктурных особенностях кристаллов $\mathrm{TlFeS}_{2}$ и $\mathrm{TlFeSe}_{2}$, которые следовало бы обнаружить методами рассеяния электромагнитного излучения (рентгеноструктурный метод, комбинационное рассеяние света, ИК-спектроскопия) в таких кристаллах, убедившись прежде всего в их однофазности.

С учетом вышеизложенного, нашей целью являлось синтезирование однофазных кристаллов $\mathrm{TlFeS}_{2}$ и $\mathrm{TlFeSe}_{2}$ и характеризация полученных кристаллов экспериментальными исследованиями и первопринципными вычислениями динамики кристаллической решетки.

\section{2. Синтез соединений и характеризация образцов}

Синтез составов $\mathrm{TlFeS}_{2}$ и $\mathrm{TlFeSe}_{2}$ осуществлялся методом высокотемпературного синтеза - сплавлением 
Таблица 1. Параметры элементарной ячейки, определенные из данных рентгеновской дифракции [12], и оптимизированные равновесные значения этих параметров из теоретических расчетов

\begin{tabular}{c|c|c|c|c}
\hline Crystals & $a, \AA$ & $b, \AA$ & $c, \AA$ & $\beta$, angle $^{\circ}$ \\
\hline \multirow{2}{*}{$\mathrm{TIFeS}_{2}$} & $11.636(28)[12]$ & $5.304(02)[12]$ & $6.799(27)[12]$ & $116.71(21)[12]$ \\
\cline { 2 - 5 } & 11.795 & 5.054 & 6.746 & 117.425 \\
\hline \multirow{2}{*}{$\mathrm{TIFeSe}_{2}$} & $11.9730(15)[12]$ & $5.4900(05)[12]$ & $7.1105(15)[12]$ & $118.17(10)[12]$ \\
\cline { 2 - 5 } & 12.156 & 5.465 & 7.302 & 118.235
\end{tabular}

химических элементов особой чистоты, взвешенных в стехиометрическом соотношении - в вакуумированных ампулах, изготовленных из плавленого кварца. Ампулы поочередно помещались в электропечь, предварительно нагретую до $\sim 1150^{\circ} \mathrm{C}$, при этом одна треть длины каждой ампулы была вне печи.

Когда часть ампулы, находящийся внутри печи, принимала ее температуру, начиналась бурно протекающая реакция (экзотермическая) образования сплава, с интенсивным свечением внутри ампулы. В процессе постепенного вхождения ампулы внутрь печи производилось медленное вращение ампулы и после полного прохождения ампулы внутрь печи, ее температура опускалась до температуры приблизительно на тридцать градусов больше температур плавления $\mathrm{TlFeS}_{2}$ и $\mathrm{TlFeSe}_{2}$ соответственно [2]. Переплавка продукта реакции длилась $72 \mathrm{~h}$, после чего вращение ампулы пресекалось и температура печи опускалась до $\sim 350^{\circ} \mathrm{C}$, при которой каждый сплав отжигался $240 \mathrm{~h}$.

Для рентгеноструктурных исследований синтезированные сплавы приводились в порошкообразное состояние, а для оптических же исследований (комбинационное рассеяние света, ИК-отражение) экспериментальные образцы были приготовлены в форме прямоугольных параллепипедов (прямоугольных призм), грани которых тщательно полировались.

\section{3. Дифракция рентгеновского излучения}

Рентгеновские порошковые дифракционные данные получены на дифрактометре „D2 Phaser", a фазовые анализы проведены методом Ритвельда с использованием стандартных программ EVA и TOPAS-4.2 (Bruker, Germany).

В интервале углов $10^{\circ} \leq 2 \theta \leq 60^{\circ}$ в кристаллах $\mathrm{TlFeS}_{2}$ и $\mathrm{TlFeSe}_{2}$ были зафиксированы характерные рентгеновские рефлексы (рис. $1, a$ и $b$ соответственно). Уточнение параметров элементарной ячейки (ЭЯ) кристаллов $\mathrm{TlFeS}_{2}$ и $\mathrm{TlFeSe}_{2}$ подтвердило, что они изоструктурны и относятся к моноклинной сингонии (табл. 1). Следует отметить, что выявленные нами значения параметров ЭЯ $\mathrm{TlFeS}_{2}$ и $\mathrm{TlFeSe}_{2}$ удовлетворительно согласуются с приведенными в работе [12].

\section{4. Комбинационное рассеяние света в соединениях $\mathrm{TIFeS}_{2}$ и $\mathrm{TIFeSe}_{2}$}

Комбинационное рассеяние света в экспериментальных образцах исследовалось на конфокальном рамановском микроспектрометре „Nanofinder 30“ (Tokyo Instr., Japan). Исследования проводились в геометрии обратного рассеяния. В качестве источника возбуждающего света использовался лазер YAG: $\mathrm{Nd}$ с длиной волны излучения на второй гармонике $\lambda=532 \mathrm{~nm}$. Приемником излучения служила охлаждаемая ССД камера $\left(-70^{\circ} \mathrm{C}\right)$, работающая в режиме счета фотонов. Время экспозиции обычно составляло $1 \mathrm{~min}$, мощность падающего на образец излучения $10 \mathrm{~mW}$, диаметр луча $4 \mathrm{mkm}$. В спектрометре использовалась дифракционная решетка 1800 lines/mm, точность определения спектрального положения линий была не хуже $0.5 \mathrm{~cm}^{-1}$. Спектры комбинационного рассеяния (KР) света в кристаллах $\mathrm{TlFeS}_{2}$ и $\mathrm{TlFeSe}_{2}$ приведены на рис. 2 ( $a$ и $b$ соответственно).

Как видно из рис. $2, a$, наблюдаются следующие частоты КР-активных фононов в кристаллах $\mathrm{TlFeS}_{2}: 47,116$, $125,163,300,354,380 \mathrm{~cm}^{-1}$.

На рис. 2, $b$ приведен спектр комбинационного рассеяния света в кристаллах $\mathrm{TlFeSe}_{2}$. Последовательно наблюдаются следующие частоты КР-активных фононов: $36.5,141,158,209 \mathrm{~cm}^{-1}$. Из сравнения частот КР-активных фононов в изоструктурных кристаллах $\mathrm{TlFeS}_{2}$ и $\mathrm{TlFeSe}_{2}$ отчетливо прослеживается, что частоты фононов кристаллов $\mathrm{TlFeSe}_{2}$ меньше частот фононов в $\mathrm{TlFeS}_{2}$, что обусловлено, по-видимому, тем, что масса атомов селена $\mathrm{Se}$ больше массы атомов серы $\mathrm{S}$.

\section{5. Инфракрасное отражение света на кристаллах $\mathrm{TIFeS}_{2}$, $\mathrm{TIFeSe}_{2}$}

Инфракрасные спектры (ИК) исследовались на инфракрасном Фурье-спектрометре Vertex70 (Bruker, Germany) в вакуумной камере с приставкой диффузного отражения, в спектральном диапазоне от 4000 до $40 \mathrm{~cm}^{-1}$, стандартное спектральное разрешение - не хуже $0.5 \mathrm{~cm}^{-1}$. Температура образцов во всех проведенных исследованиях, как и в случае исследований комбинационного рассеяния света, была равной $300 \mathrm{~K}$. 

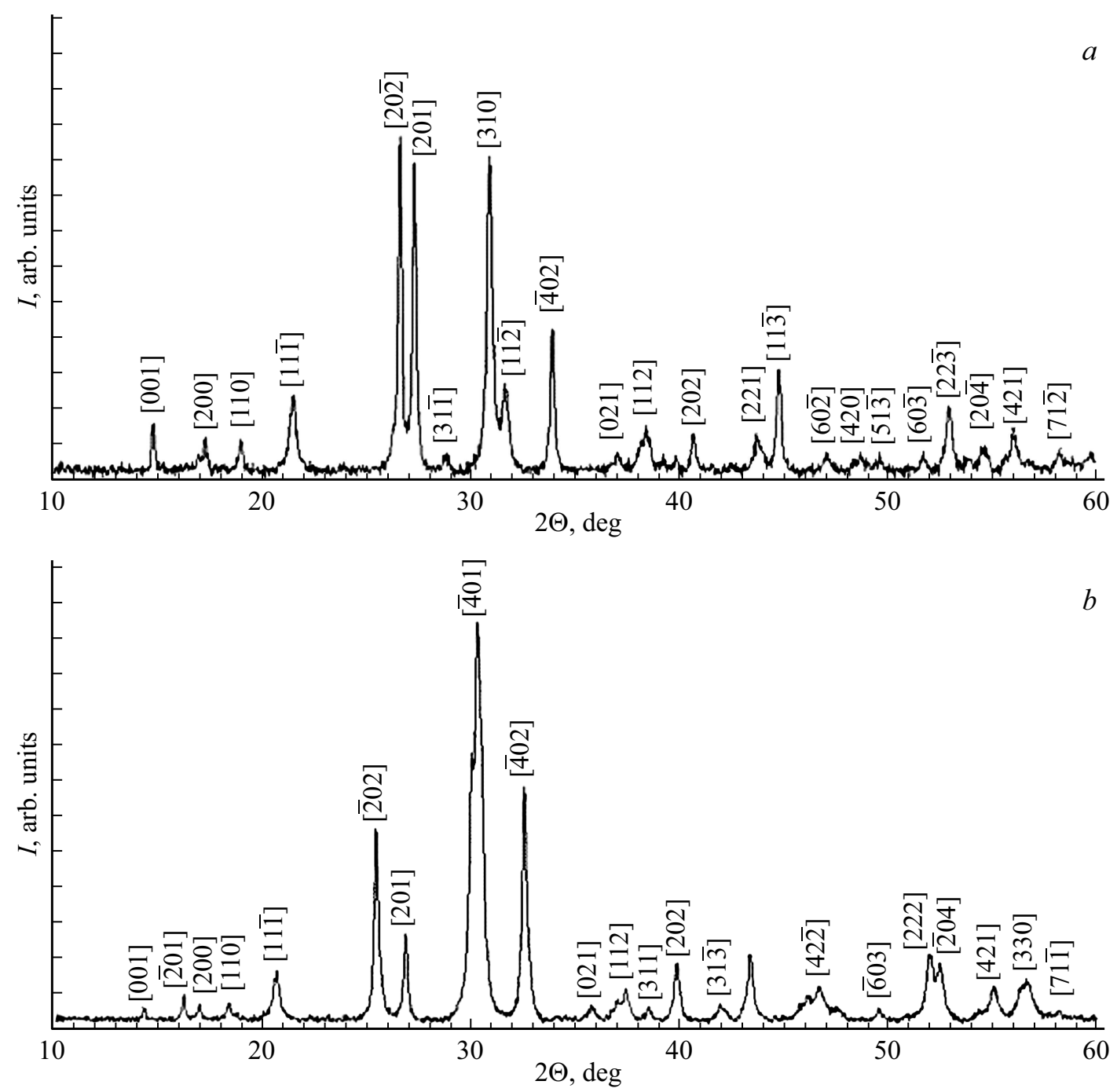

Рис. 1. Рентгеновские дифрактограммы кристаллов $\mathrm{TlFeS}_{2}(a)$ и $\mathrm{TlFeSe}_{2}(b)$.
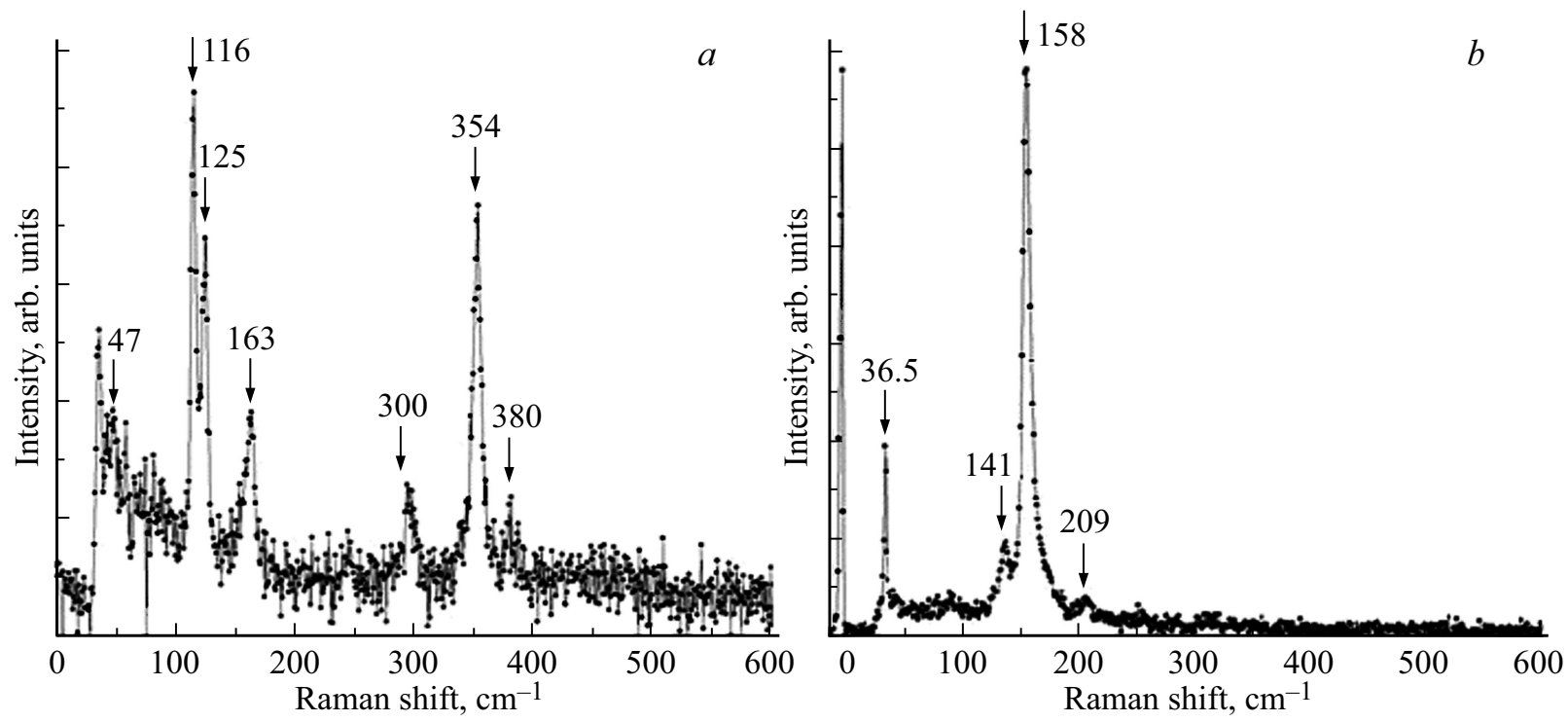

Рис. 2. Спектры комбинационного рассеяния света в кристаллах $\mathrm{TlFeS}_{2}(a)$ и $\mathrm{TlFeSe}_{2}(b)$. 

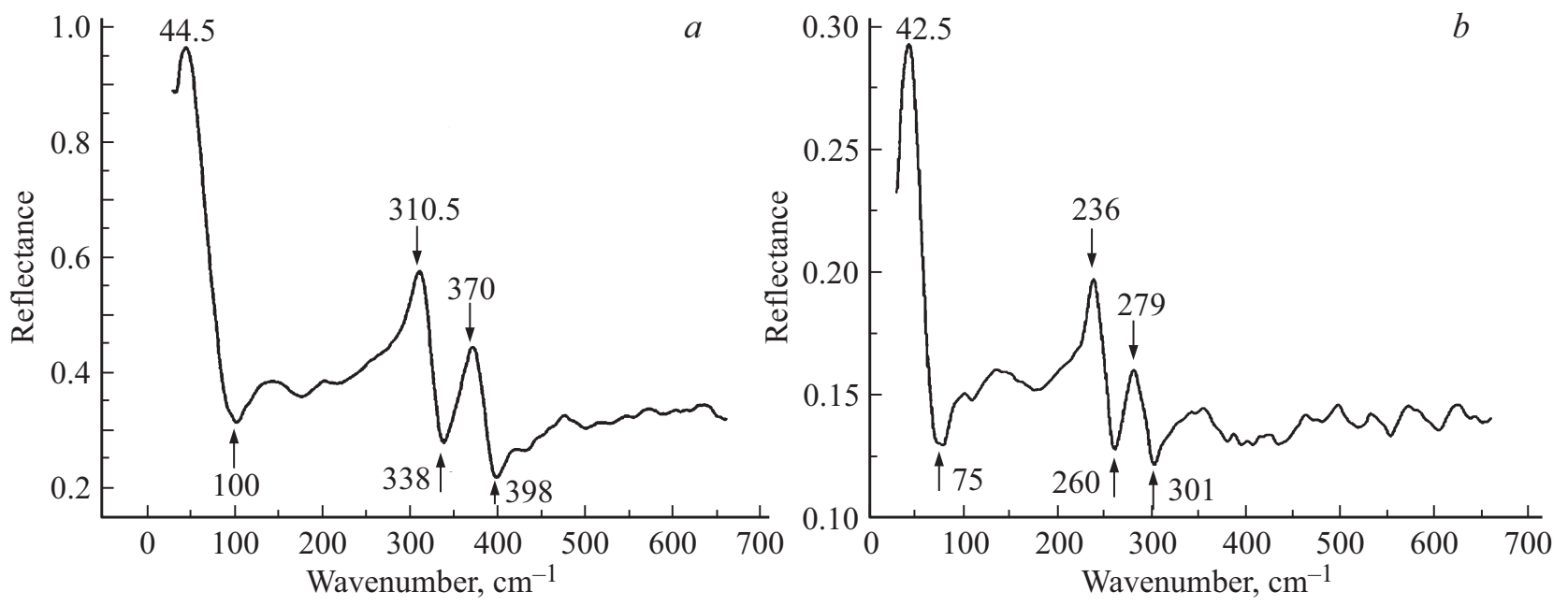

Pис. 3. Спектры инфракрасного отражения света в кристаллах $\mathrm{TlFeS}_{2}(a)$ и $\mathrm{TlFeSe}_{2}(b)$.

На рис. 3, $a$ приведен спектр ИК-отражения света на кристаллах $\mathrm{TlFeS}_{2}$. Отражение света происходило под углом 45 градусов. Из рис. 3, $a$ видны особенности спектра отражения, обусловленные наличием характерных ИК-активных фононов с частотами: $72,324,384 \mathrm{~cm}^{-1}$.

На рис. 3, $b$ приведен спектр ИК-отражение света на кристаллах $\mathrm{TlFeSe}_{2}$, из которого видны особенности спектра отражения, обусловленные наличием характерных ИК-активных фононов с частотами: 59,248 , $290 \mathrm{~cm}^{-1}$. Отметим, что и как в случае со спектрами комбинационного рассеяния света, величины ИК-активных частот кристаллов $\mathrm{TlFeSe}_{2}$ меньше частот фононов в $\mathrm{TlFeS}_{2}$, что также обусловлено, по-видимому, тем, что масса атомов селена Se больше массы атомов серы S. Следует еще отметить, что спектры ИК-отражения света на кристаллах $\mathrm{TlFeS}_{2}$ и $\mathrm{TlFeSe}_{2}$ имеют очень похожий вид, и тем самым косвенно подтверждается изоструктурность этих соединений.

\section{Ab initio расчеты колебательных спектров кристаллов $\mathrm{TIFeS}_{2}, \mathrm{TIFeSe}_{2}$}

Ранее нами были рассчитаны электронная структура и плотность состояний $\mathrm{TlFeSe}_{2}$ и $\mathrm{TlFeS}_{2}$ [13], а также расчеты ab initio дисперсии фононов в халькопиритах $\mathrm{CdGa}_{2} \mathrm{~S}_{4}$ [14] и $\mathrm{CdGa}_{2} \mathrm{Te}_{4}$ [15]. Ниже представлены результаты ab initio расчетов дисперсии фононов и плотности фононных состояний в высокосимметричных точках и линиях зоны Бриллюэна (ЗБ) кристаллов $\mathrm{TlFeSe}_{2}$ и $\mathrm{TlFeS}_{2}$. Как нам известно, в литературе отсутствуют первопринципные расчеты фононного спектра $\mathrm{TlFeSe}_{2}$ и $\mathrm{TlFeS}_{2}$.

Ab-initio расчеты динамических характеристик проводились с помощью теории возмущений функционала плотности DFPT (Density Functional Perturbation Theory) [16-18] с использованием метода псевдопотенциала на основе плоских волн, реализованного в коде
ABINIT [19]. В качестве псевдопотенциалов использовались сохраняющие нормы псевдопотенциалы HartwigsenGoedecker-Hutter (HGH) [20]. Обменно-корреляционное взаимодействие описывалось в обобщенном градиентном приближении (GGA) [21]. В разложении волновых функций были учтены плоские волны с максимальной кинетической энергией до $80 \mathrm{Ry}$, которые обеспечивают хорошую сходимость полной энергии. Численное интегрирование по зоне Бриллюэна (ЗБ) проводилось с помощью разбиения $4 \times 4 \times 4$ со сдвигом $(0.5,0.5,0.5)$ от начала координат, согласно схеме Монкхорста-Пака [22]. Параметры решетки и равновесные положения атомов в элементарной ячейке определялись минимизацией сил Геллмана-Фейнмана (табл. 1). При этом равновесные положения атомов внутри элементарной ячейки кристалла были найдены методом BFGS (Broyden-FletcherGoldfarb-Shanno) с использованием экспериментальных данных в качестве начальных значений. Процедура минимизации проводилась до тех пор, пока не достигалось значение модуля сил, меньше 10-8 Ry/Bohr. C помощью $40 \times 40 \times 40-$ разбиения ЗБ определена фононная плотность состояний. Для получения LO-TO (продольные и поперечные оптические фононы) расщепления в центре ЗБ при полярных модах, учтено дальнодействующее кулоновское поле и в динамическую матрицу добавлен неаналитический член, который зависит от тензоров эффективного заряда Борна и электронной диэлектрической проницаемости. Зависимость сходимости полной энергии и сил Геллмана-Фейнмана от сетки Монхорста-Пака и от максимальной энергии плоских волн с учетом оптимальной употребляемой машинной времени для вычислений показали, что сетка $4 \times 4 \times 4$ и максимальная энергия плоских волн $80 \mathrm{Ry}$ в разложении волновых функций дают достаточно хорошие результаты для описания электронных и динамических свойств $\mathrm{TlFeS}_{2}$ и $\mathrm{TlFeSe}_{2}$.

B примитивной ячейке исследуемых соединений имеется 8 атомов, соответственно колебательный 


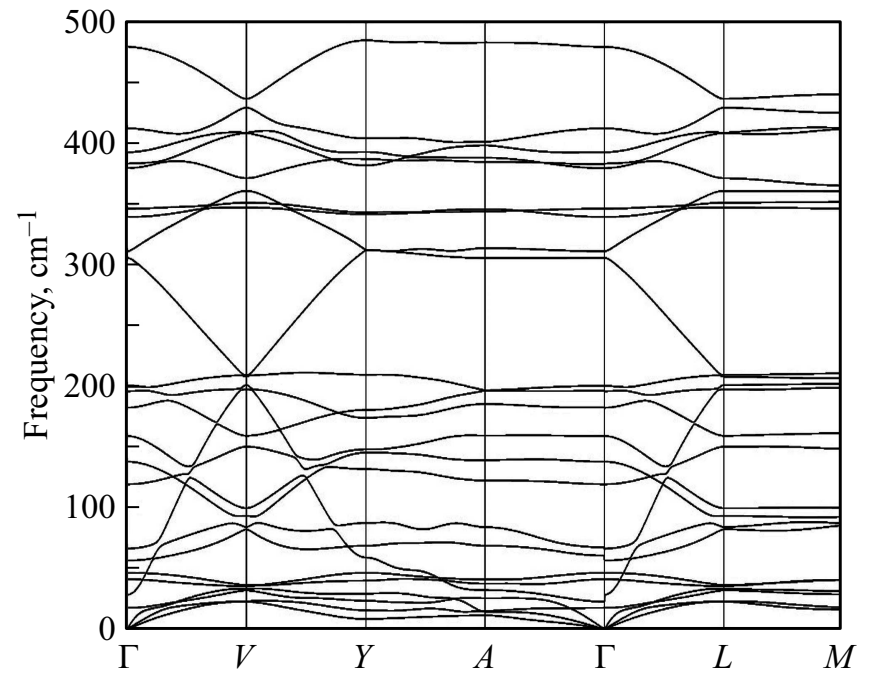

Рис. 4. Дисперсия фононов в $\mathrm{TlFeS}_{2}$.

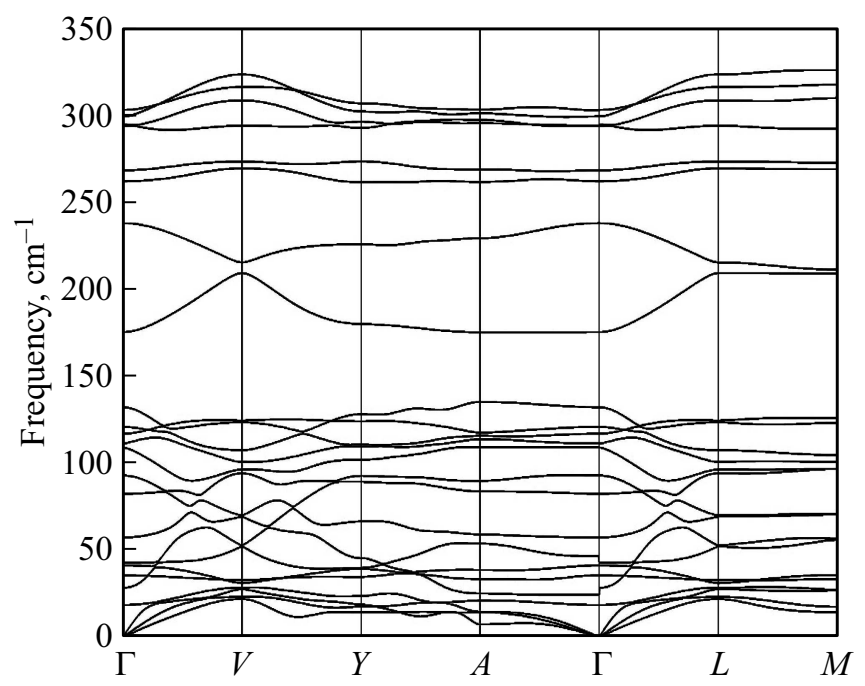

Рис. 5. Дисперсия фононов в $\mathrm{TlFeSe}_{2}$.

спектр состоит из 24 нормальных мод и описывается следующими неприводимыми представлениями: $\Gamma=6 \mathrm{~A}_{\mathrm{g}}+4 \mathrm{~A}_{\mathrm{u}}+6 \mathrm{~B}_{\mathrm{g}}+8 \mathrm{~B}_{\mathrm{u}}$, акустические моды $\Gamma_{\text {acoustic }}=$ $=\mathrm{A}_{\mathrm{u}}+2 \mathrm{~B}_{\mathrm{u}} \quad$ и оптические моды $\Gamma_{\text {optic }}=6 \mathrm{~A}_{\mathrm{g}}+3 \mathrm{~A}_{\mathrm{u}}$ $+6 \mathrm{~B}_{\mathrm{g}}+6 \mathrm{~B}_{\mathrm{u}}$.

Нормальные моды $\mathrm{A}_{\mathrm{g}}$ и $\mathrm{B}_{\mathrm{g}}$ симметрии активны в комбинационном рассеянии (КР), а моды $\mathrm{A}_{\mathrm{u}}$ и $\mathrm{B}_{\mathrm{u}}$ активны в спектрах инфракрасного (ИК) поглащения и отражения. Вследствие наличия центра симметрии, действует правило альтернативного запрета и в КР не могут проявляться ИК-активные моды.

На рис. 2, $a$ и $b$ представлены спектры комбинационного рассеяния и ИК-отражения, соответственно для $\mathrm{TlFeS}_{2}$ и $\mathrm{TlFeSe}_{2}$. Результаты ab-initio расчетов фононных мод были использованы для идентифицирования этих колебаний. На рис. 4 и 5 показаны дисперсия фононных мод $\mathrm{TlFeS}_{2}$ и $\mathrm{TlFeSe}_{2}$ вдоль высокосиммет- ричных линий ЗБ. ИК активные $\mathrm{A}_{u}$ и $\mathrm{B}_{\mathrm{u}}$ моды вызывают LO-TO расщепление в центре ЗБ и видны как разрывы в спектре (рис. 4, 5). Сравнение фононных спектров изоструктурных соединений $\mathrm{TlFeS}_{2}$ и $\mathrm{TlFeSe}_{2}$ показывает, что характер дисперсии фононов в общем совпадают. Только, с уменьшением атомной массы аниона значения максимума оптических мод увеличивается. Проектированные на атомы плотности фононных состояний (PDOS) показаны на рис. 6 и 7. Анализ собственных векторов для каждого атома в примитивной ячейке и $\mathrm{PDOS}$ показывает, что акустические и низкочастотные оптические ветви в частотном интервале от 0 до $60 \mathrm{~cm}^{-1}$ с максимумом при $\sim 35 \mathrm{~cm}^{-1}$ в основном, связаны с колебанием атома $\mathrm{Tl}$, с небольшим вкладом атомов $\mathrm{Fe}$ и аниона для обоих кристаллов. Второй частотный интервал для $\mathrm{TlFeS}_{2}\left(\mathrm{TlFeSe}_{2}\right)$ от 60

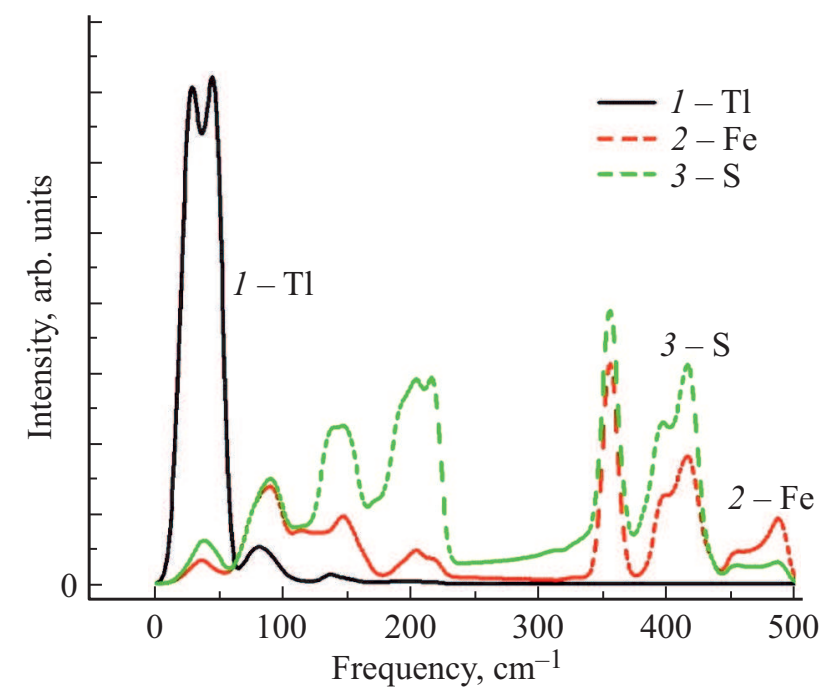

Рис. 6. Проектированный на атомы плотности фононных состояний в $\mathrm{TlFeS}_{2}$.

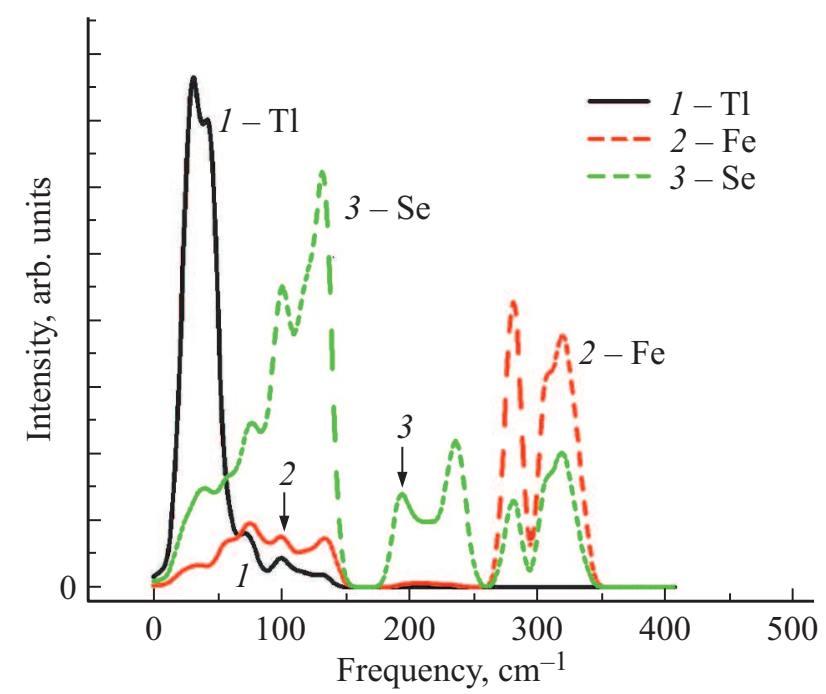

Рис. 7. Проектированный на атомы плотности фононных состояний в $\mathrm{TlFeSe}{ }_{2}$. 
Таблица 2. Экспериментально определенные и вычисленные с учетом макроскопического электрического поля в направлении [100] (в декартовых координатах) частоты оптических фононов в $\mathrm{TlFeS}_{2}$ и $\mathrm{TlFeSe}_{2}$

\begin{tabular}{|c|c|c|c|c|c|c|}
\hline \multicolumn{4}{|c|}{$\mathrm{TlFeSe}_{2}$} & \multicolumn{3}{|c|}{$\mathrm{TlFeS}_{2}$} \\
\hline \multirow{2}{*}{ Mode } & \multirow{2}{*}{$\begin{array}{c}\omega_{\mathrm{th}}, \mathrm{cm}^{-1}, \\
\text { theory }\end{array}$} & \multicolumn{2}{|c|}{$\omega_{\exp }, \mathrm{cm}^{-1}$} & \multirow{2}{*}{$\begin{array}{c}\omega_{\mathrm{th}}, \mathrm{cm}^{-1}, \\
\text { theory }\end{array}$} & \multicolumn{2}{|c|}{$\omega_{\mathrm{exp}}, \mathrm{cm}^{-1}$} \\
\hline & & IR & $\mathrm{R}$ & & IR & $\mathrm{R}$ \\
\hline $\operatorname{Ag}(\mathrm{R})$ & $\begin{array}{r}35.5 \\
41.2 \\
82.4 \\
148.5 \\
223.1 \\
299.3\end{array}$ & & $\begin{array}{l}36.5 \\
\\
158 \\
209\end{array}$ & $\begin{array}{r}41.2 \\
46.5 \\
119.4 \\
200.4 \\
311.3 \\
479.6\end{array}$ & & $\begin{array}{r}47 \\
116 \\
300\end{array}$ \\
\hline $\mathrm{Bg}(\mathrm{R})$ & $\begin{array}{r}18.5 \\
92.9 \\
109.1 \\
133.7 \\
262.1 \\
268.3\end{array}$ & & 141 & $\begin{array}{r}17.6 \\
138.0 \\
159.2 \\
339.6 \\
346.4 \\
412\end{array}$ & & $\begin{array}{l}125 \\
163 \\
354\end{array}$ \\
\hline $\mathrm{Au}(\mathrm{IR})$ & $\begin{array}{r}24.3 \\
175.3 \\
273.8\end{array}$ & 248 & & $\begin{array}{r}22.7 \\
316.0 \\
383.0\end{array}$ & 324 & 380 \\
\hline $\mathrm{Bu}(\mathrm{IR})$ & $\begin{array}{r}42.5 \\
56.8 \\
111.3 \\
116.9 \\
293.8 \\
302.4\end{array}$ & 290 & & $\begin{array}{r}56.6 \\
65.8 \\
182.4 \\
196.1 \\
379.6 \\
392.9\end{array}$ & 384 & \\
\hline
\end{tabular}

до $240 \mathrm{~cm}^{-1}\left(150 \mathrm{~cm}^{-1}\right)$ с максимумом при $218 \mathrm{~cm}^{-1}$ $\left(133 \mathrm{~cm}^{-1}\right)$ в основном, связан с колебанием атома $\mathrm{S}(\mathrm{Se})$, с небольшим вкладом Fе. В этом интервале атомы $\mathrm{Tl}$ в фононных колебаниях почти не участвуют. В частотном интервале $178-258 \mathrm{~cm}^{-1}$ для кристалла $\mathrm{TlFeSe}$ в колебаниях участвует только атомы Se. Аналогичный частотный интервал для $\mathrm{TlFeS}_{2}$ отсутствует. Высокочастотный интервал $338-500 \mathrm{~cm}^{-1}$ и $260-335 \mathrm{~cm}^{-1}$ для $\mathrm{TlFeSe}_{2}$ и $\mathrm{TlFeS}_{2}$, соответственно, состоят из колебаний $\mathrm{Fe}$ и атомов аниона. Надо отметить, что атомы $\mathrm{Tl}$ участвуют в колебаниях только в частотном интервале до $100 \mathrm{~cm}^{-1}$. В средних и высоких частотах их вклад почти отсутствует. Кристаллоструктура изоструктурных соединений $\mathrm{TlFeS}_{2}$ и $\mathrm{TlFeSe}_{2}$ обладает двумя структурными построениями: тетраэдрическими $\mathrm{Fe} X_{4}(X-$ атом халькогена, $\mathrm{S}, \mathrm{Se})$ цепочками и подрешеткой со слоевыми пакетами из атомов Tl. Между слоями действуют слабые связи ван-дер-ваальсового типа, поэтому атомы Tl фактически участвуют лишь в низкочастотной динамике кристаллической решетки $\mathrm{TlFeS}_{2}, \mathrm{TlFeSe}_{2}$.

Наиболее интенсивный пик в спектрах КР при 116 и $158 \mathrm{~cm}^{-1}$ для $\mathrm{TlFeSe}_{2}$ и $\mathrm{TlFeS}_{2}$, соответственно, соответствует $\mathrm{A}_{\mathrm{g}}$ моде, в колебаниях которых участвуют атомы $\mathrm{Fe}, \mathrm{S}$ и $\mathrm{Se} . \mathrm{B}$ табл. 2 приведены рассчитанные фононные частоты с учетом макроскопического электрического поля (с направлением [100] в декартовых координатах) и экспериментальные данные исследований ИК- и КР-спектров $\mathrm{TlFeSe}_{2}$ и $\mathrm{TlFeS}_{2}$. Появление слабоинтенсивной ИК-моды $380 \mathrm{~cm}^{-1}$ в спектре КР кристалла $\mathrm{TlFeS}_{2}$, по-видимому, обусловлено нарушением симметрии вследствие дефектности структуры.

\section{7. Заключение}

На основе расчетов из первых принципов в рамках теории функционала плотности выполнен расчет колебательных спектров и плотности фононных состояний кристаллов $\mathrm{TlFeSe}_{2}$ и $\mathrm{TlFeS}_{2}$ с моноклинной структурой кристаллической решетки. Результаты расчетов показывают, что при переходе от S-содержащего соединения к Se-содержащему происходит понижение частот верхних колебательных мод, что связано с увеличением массы аниона. При этом, характер дисперсии фононов в общем совпадает. Из экспериментальных исследований комбинационного рассеяния света в геометрии обратного рассеяния выявлены следующие частоты КР-активных фононов в кристаллах $\mathrm{TlFeS}_{2}: 47,116,125,163,300,354$, $380 \mathrm{~cm}^{-1}$ и частоты КР-активных фононов кристаллах $\mathrm{TlFeSe}_{2}$ : 36.5, 141, 158, $209 \mathrm{~cm}^{-1}$. Появление слабоинтенсивной ИК-моды $380 \mathrm{~cm}^{-1}$ в спектре КР кристалла $\mathrm{TlFeS}_{2}$, по-видимому, обусловлено нарушением симметрии вследствие наличия дефектов структуры. Из данных изучения ИК-отражения света определены частоты ИК активных фононов в кристаллах $\mathrm{TlFeS}_{2}(72,324$, $\left.384 \mathrm{~cm}^{-1}\right)$ и $\mathrm{TlFeSe}_{2}\left(59,248,290 \mathrm{~cm}^{-1}\right)$. Полученные расчетные результаты хорошо согласуются с экспериментальными данными из КР- и ИК-спектров кристаллов $\mathrm{TlFeSe}_{2}$ и $\mathrm{TlFeS}_{2}$. Планируется провести исследования магнитной восприимчивости соединений $\mathrm{TlFeS}_{2}, \mathrm{TlFeSe}_{2}$ в интервале температур $0.5-400 \mathrm{~K}$.

\section{Благодарности}

Авторы выражают благодарность А.С. Бондякову (ОИЯИ, Россия) и Д.А. Кулиеву (Институт физики НАН Азербайджана), а также всему коллективу Дата-центра Института физики НАН Азербайджана, за предоставленные ресурсы и техническую поддержку теоретических расчетов.

\section{Финансирование работы}

Работа выполнена при финансовой поддержке Фонда развития науки при Президенте Азербайджанской республики (гранты № EİF/MQM/Elm-Tehsil-1-2016-1(26)71/16/1 и № EİF-BGM-3-BRFTF-2+/2017-15/02/1).

\section{Конфликт интересов}

Авторы заявляют, что у них нет конфликта интересов. 


\section{Список литературы}

[1] D. Welz, P. Deppe, W. Schafer, H. Sabrowsky, M. Rosenberg. Phys. Chem. Solids 50, 3, 297 (1989).

[2] Г.И. Маковецкий, Е.И. Касинский. Изв. АН СССР. Неорган. материалы 20, 10, 1752 (1984).

[3] M.A. Aldzhanov, N.G. Guseinov, G.D. Sultanov, M.D. Nadzafzade. Phys. Status SolidiB 159(2), K107 (1990).

[4] H. Sabrowsky, M. Rosenberg, D. Welz, P. Deppe, W. Schafer. J. Magn. Magn. Mater. 54-5, 3, 1497 (1986).

[5] Г.Д. Султанов, Г.Д. Гусейнов, С.Г. Ибрагимов, А.С. Шукюров. Докл. АН АзССР, 43, 2, 16 (1987).

[6] Г.Д. Султанов, Р.М. Мирзабабаев, С.Г. Ибрагимов, А.С. Шукюров, М.Т. Касумов. ФТТ 29, 7, 2138 (1987).

[7] Э.М. Керимова, Ф.М. Сеидов, С.Н. Мустафаева, С.С. Абдинбеков. Изв. РАН, Неорган. материалы 35, 2, 157 (1999).

[8] Z. Seidov, H.-A. Krug von Nidda, J. Hemberger, A. Loidl, G. Sultanov, E. Kerimova, A. Panfilov. Phys. Rev. B 65, 1, 014433 (2001).

[9] Э.Б. Аскеров, А.И. Мададзада, А.И. Бескровный, Д.И. Исмаилов, Р.Н. Мехдиева, С.Г. Джабаров, Э.М. Керимова, Д. Неов. Поверхность, Рентгеновские, синхротронные и нейтронные исследования 12, 1 (2014).

[10] Z. Seidov, H.-A. Krug von Nidda, V. Tsurkan, I. Filippova, A. Günther,A. Najafov, M.N. Aliyev, F.G. Vagizov, A.G. Kilamov, L.R. Tagirov, T. Gavrilova, A. Loidl. Bull. RAS: Phys. 81, 7, 885 (2017).

[11] Р.Г. Велиев. ФТП 45, 2, 162 (2011).

[12] K. Klepp, H. Boller, Monatsh. Chem. 110 (1979) 1045 (in German).

[13] K. Mimura, S. Motonami, Y. Shim, K. Wakita, Z. Jahangirli, O. Alekperov, N. Mamedov, H. Sato, Y. Utsumi, S. Ueda, K. Shimada, Y. Taguchi, K. Kobayashi, G. Bihlmayer, H. Namatame, M. Taniguci. Phys. Status Solidi C 10, 7-8, 989 (2013).

[14] 3.А. Джахангирли, Т.Г. Керимова, И.А. Мамедова, Н.А. Абдуллаев, Н.Т. Мамедов. ФТТ 60, 11, 2265 (2018).

[15] З.А. Джахангирли, Т.Г. Керимова, И.А. Мамедова, С.А. Набиева, Н.А. Абдуллаев. ФТТ 62, 8, 1270 (2020).

[16] P. Gianozzi, S. deGironcoli, P. Pavone, S. Baroni. Phys. Rev. B 43, 7231 (1991).

[17] S. Baroni, S. deGironcoli, A. Dal Corso, P. Gianozzi. Rev. Mod. Phys. 73, 515 (2001).

[18] X. Gonze. Phys. Rev. B 55, 10337 (1997).

[19] X. Gonze, J.M. Beuken, R. Caracas, F. Detraux, M. Fuchs., G.M. Rignanese, L. Sindic, M. Verstraete, G. Zerah, F. Jallet. Comput. Mater. Sci. 25, 478 (2002).

[20] C. Hartwigsen, S. Goedecker, J. Hutter. Phys. Rev. B 58, 3641 (1998).

[21] J.P. Perdew, A. Zunger. Phys. Rev. B 23, 5048 (1981).

[22] H. Monkhorst, J. Pack. Phys. Rev. B 13, 5188 (1976).

\section{Редактор К.В. Емщев}

